\title{
Evidence of Conducting Hydrophobic Nanopores Across Membranes in Response to an Electric Field
}

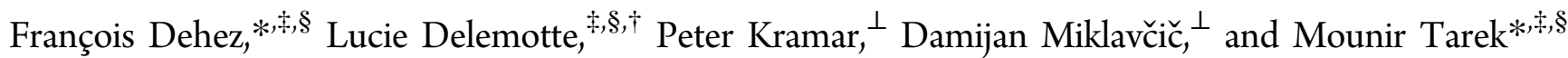 \\ ¥CNRS, UMR 7565, SRSMC, F-54506 Vandœuvre-lès-Nancy, France \\ ${ }^{\S}$ Université de Lorraine, UMR 7565, SRSMC, F-54506 Vandœuvre-lès-Nancy, France \\ ${ }^{\perp}$ Faculty of Electrical Engineering, University of Ljubljana, Ljubljana, Slovenia
}

Supporting Information

\begin{abstract}
Electroporation, the application of electric fields to alter the permeability of biological membranes, has recently become a clinical tool for the electrochemotherapy treatment of various cancers. Current electroporation theory assumes that the membrane is permeabilized through the formation of conducting hydrophilic pores, stabilized by rearrangement of lipid head groups. Here we have performed molecular dynamics simulations of negatively charged lipid bilayers subject to high transmembrane voltages together with electroporation experiments on planar bilayers. Our data reveal a hitherto unknown electroporation process in which large ion-conducting water columns not stabilized by lipid head groups are formed within the bilayer's hydrophobic core. The existence of such hydrophobic pores challenges the standard theoretical description of pore creation in lipid membranes. Our findings open a new vista toward fine-tuning of electroporation-based treatments and biotechnical applications, and, in general, for enhancing the import of various substrates in liposomes or cells.
\end{abstract}

\section{INTRODUCTION}

Electroporation (EP) is a process in which lipid membranes, the cell envelopes, are permeabilized when subjected to high enough electric fields. ${ }^{1}$ The technique also known as electropermeabilization $^{2,3}$ is nowadays widely used in various applications ranging from in vitro DNA and siRNA cell delivery $^{4,5}$ to clinical electrochemotherapy where delivery of drugs to cancer cells is enhanced. ${ }^{6-8}$ Experimental evidence suggests that the electric fields act primarily in the lipid domain of the membrane, and the key features of EP are based on theories involving the formation of stochastic pores. ${ }^{9}$ In erythrocyte membranes, large pores could be observed by using electron microscopy, ${ }^{10}$ but in general, direct observation of nanosized pores is not possible with conventional techniques. It is, however, largely accepted that high transmembrane (TM) voltages produce aqueous-filled pores in the lipid bilayer ${ }^{10-14}$ as evidenced by early molecular-dynamics simulation ${ }^{15,16}$ and recent experiments. ${ }^{17,18}$ The properties of pores, e.g., size, density, and lifetime (after the electric pulse shutdown), are key factors that determine how leaky a membrane can become. ${ }^{19}$

Traditionally, electroporation is triggered by applying long (microseconds) electric pulses of low magnitude $(\mathrm{kV} / \mathrm{m})$. The resulting current causes an accumulation of electrical charges at both sides of the cell membrane over a time that depends on the electrical parameters of the system, often in the order of 100 s of $\mathrm{ns}^{20-24}$ As the membrane behaves as a capacitor, this charge accumulation gives rise to a TM voltage. Simulation protocols have so far been developed to mimic in silico the effect of low magnitude microsecond pulses (msEP) on planar bilayers $^{25}$ by imposing a net charge imbalance across the membrane. Such a protocol has been used to monitor the effect of high voltages on zwitterionic membranes. ${ }^{26-31}$ The simulations show that it triggers rearrangements of the membrane components, i.e., water and lipids. When the TM voltage is above a threshold value characteristic of the lipid composition, the membrane loses its integrity. This enables the ionic and molecular transport through the otherwise impermeable membranes to increase substantially. ${ }^{18}$ Electroporation starts with the formation of water fingers that protrude inside the hydrophobic core of the membrane. Within nanoseconds, water wires bridging the two sides of the lipid bilayer appear. If the simulations are further extended, lipid head groups migrate along one wire and form a hydrophilic connected pathway and if voltage is maintained, ions start to flow through this pathway. Moreover, when the TM voltage is lowered, or is reduced due to ionic flow across the membrane, the pores collapse to reach a nonsolvated and nonconductive state. ${ }^{25,27-29}$ It was shown that full recovery of the membrane integrity (migration of the lipid head groups back to the lipid water interface) requires few tens of nanoseconds. ${ }^{29,32}$

Although a considerable effort has been devoted to characterize basic processes of classical electroporation at a molecular level better, the details of the phenomenon in

Received: November 22, 2013

Revised: February 21, 2014

Published: February 24, 2014 
membranes of increasing complexity still remain to be elucidated. Indeed, to date, most simulations have been performed on membranes formed by zwitteroinic lipids (phosphatidyl choline (PC) head groups) with the exception of a few that considered for instance the presence of negatively charged lipids to study their externalization, ${ }^{31,33-35}$ a cholesterol fraction, ${ }^{36}$ or Escherichia coli and Staphylococcus aureus model membranes. ${ }^{37}$ Membranes which are formed by a variety of lipids can form several phases, with domains of various compositions. Today we still lack information about the response of such membranes to external electrical stress such as that leading to electroporation. This is precisely what this study aims at. We characterize here in particular electroporation of lipid bilayers formed by negatively charged lipids.

\section{COMPUTATIONAL DETAILS}

We considered a fully hydrated palmitoyl-oleyl-phosphatidylserine (POPS) lipid bilayer embedded in a $\sim 250 \mathrm{mM} \mathrm{NaCl}$ solution. The system consisted of 392 lipids, 39200 water molecules, 576 sodium and 184 chloride ions (a total of 144 624 atoms). The bilayer was first replicated in all three dimensions and equilibrated at constant pressure (1 atm) and constant temperature $(300 \mathrm{~K})\left(103 \times 100 \times 160 \AA^{3}\right)$. At the temperature set for the study, i.e., $300 \mathrm{~K}$, the bilayer is in the biologically relevant liquid crystal $\mathrm{L} \alpha$ phase. The area per lipid reached at equilibration was $\sim 52.5 \AA^{2}$, close to the $55 \AA^{2}$ estimated for a POPS bilayer using a different force field. ${ }^{38}$

The MD simulations presented here were carried out with use of the program NAMD targeted for massively parallel architectures. $^{39}$ The systems were examined in the NPT ( $1 \mathrm{~atm}$ and $300 \mathrm{~K})$ or NVT $(300 \mathrm{~K})$ ensembles employing the Langevin dynamics and Langevin piston method. The equations of motion were integrated by using a multiple time step algorithm. A time step of $2.0 \mathrm{fs}$ was employed. Short- and long-range forces were calculated every 1 and 2 time steps, respectively. Chemical bonds between hydrogen and heavy atoms were constrained to their equilibrium value. Long-range, electrostatics forces were taken into account by using a fast implementation of the particle mesh Ewald (PME) approach, ${ }^{40,41}$ with a direct space sum tolerance of $10^{-6}$ and a spherical truncation of $11 \AA$. The water molecules were described by using the TIP3P model $^{42}$ and a united-atom representation was adopted for the acyl chains of the POPS lipid molecules ${ }^{43}$ while keeping intact the CHARMM27 description of the head groups.

The system was first equilibrated at constant temperature $(300 \mathrm{~K})$ and constant pressure (1 atm), as in the simulations of multilamellar stacks of lipids. The simulation box was then extended in the $z$ direction perpendicular to the bilayer (to 360 $\AA$ ) in order to create air (vacuum)-water interfaces, enabling a charge imbalance $(\Delta Q)$ between the solutions on either side of the membrane to be imposed. ${ }^{25,44}$ Including a vacuum slab between replicas (use of $3 \mathrm{D}$ boundary conditions) along the membrane normal in order to prevent communication between baths is an alternative workaround to the more costly double bilayer setup (see the Supporting Information (SI), Figure S1). ${ }^{26}$ As far as water slabs that are thicker than $25-30 \AA$, the presence of air water interfaces has no incidence on the lipid bilayer properties and the membrane "feels" as if it is embedded in infinite baths whose characteristics are those of the modeled finite solutions. In such a setup, the simulation is carried out at constant volume, and therefore a surface tension builds in as the bilayer undergoes electroporation.
The charge imbalance setup enables generating a transmembrane voltage by imposing a charge imbalance between the solutions on either side of the POPS bilayer. Such protocols aim at mimicking the effects of low-field pulses of microsecond or millisecond duration applied to this cell that result in an accumulation of electrical charges at both sides of the cell membrane. Note that here the "charging" of the membrane is not modeled. Rather, the simulations are initiated by assuming that the charging has already taken place, i.e., with a system already set at a specific (selected) charge imbalance. A charge imbalance $\Delta Q$ between the two sides of the bilayer is generated by simply displacing at time $t=0$ an adequate number of ions from one side to the other.

Eventually, a set of MD simulations was carried out at varying transmembrane voltages (see the SI, Table S1). The lower voltage, giving rise to electroporation on a time scale accessible to MD simulations, amounts to $4 \mathrm{~V}$. Statistics were thus gathered specifically at the threshold voltage of $4 \mathrm{~V}$ (10 simulations of 20 to $40 \mathrm{~ns}$ ). Three simulations were also conducted at higher TM voltages (see the SI, Table S1).

\section{EXPERIMENTAL METHODS}

The salt solution consists of $0.1 \mathrm{M} \mathrm{KCl}$ and $0.01 \mathrm{M}$ Hepes in the same proportion. $\mathrm{NaOH}(1 \mathrm{M})$ was added to obtain a neutral $\mathrm{pH}$ (7.4). POPS (1-pamitoyl 2-oleoyl phosphatidylserine) lipids in powder form (Avanti Polar-Lipids Inc. USA) were melted in a 9:1 hexane/ethanol solution. A 3:7 mixture of hexadecane and pentane was used for torus forming.

The chamber where planar lipid bilayers are formed consists of two $5.3 \mathrm{~cm}^{3}$ Teflon reservoirs separated by a thin Teflon sheet with a $117 \mu \mathrm{m}$ diameter round aperture ${ }^{45}$ on which bilayers are built by using the Montal-Muller method. ${ }^{46}$ The measuring system ${ }^{47}$ was comprised of four $\mathrm{Ag}$ - $\mathrm{AgCl}$ electrodes ( 2 current and 2 reference electrodes) and consisted of two modules allowing the measurement of the lipid bilayer capacitance and the voltage across the bilayer, and a potentiostat-galvanostat for the current control. The specific membrane (bilayer) capacitance $\left(c_{\mathrm{BLM}}\right)$ normalized to the surface area of the bilayer was measured with capacitance to period conversion. ${ }^{17}$ Current clamp measurements were performed by using a measuring system, as described by Kalinowski et al. ${ }^{48,49}$ and by using four $\mathrm{Ag}$-AgCl electrodes (two current electrodes and two reference electrodes). The measuring system consists of two modules. The first is a capacity to period converter, used for measuring the bilayer's capacitance. The second is a potentiostat-galvanostat for current clamp planar lipid bilayer studies. Both modules were controlled with a personal computer.

Measuring protocols consist of two parts: capacitance measurement and lipid bilayer breakdown voltage measurement. The membrane capacitance was measured with capacitance to period converting measuring principle described in detail by Kalinowski et al. and then normalized to the surface area of the bilayer to calculate the specific capacitance $\left(c_{\mathrm{BLM}}\right)$. We then determined breakdown voltage $\left(U_{\mathrm{br}}\right)$ of each lipid bilayer by applying linear rising current signals (of slope $k$ ) (see the SI, Figure S2). Six different slopes were selected: 0.5, 1, 4, 8, $10,20 \mu \mathrm{A} / \mathrm{s}$. $U_{\mathrm{br}}$ was defined as the voltage at $t_{\mathrm{br}}$, when a sudden drop in the voltage was detected (see the SI, Figure S2). Voltage breakdown $U_{\mathrm{br}}$ was measured for 34 planar lipid bilayer constructs (see the SI, Table S2). The average membrane capacitance was $0.41 \pm 0.13 \mu \mathrm{F} / \mathrm{cm}^{2}$. Voltage drops such as 

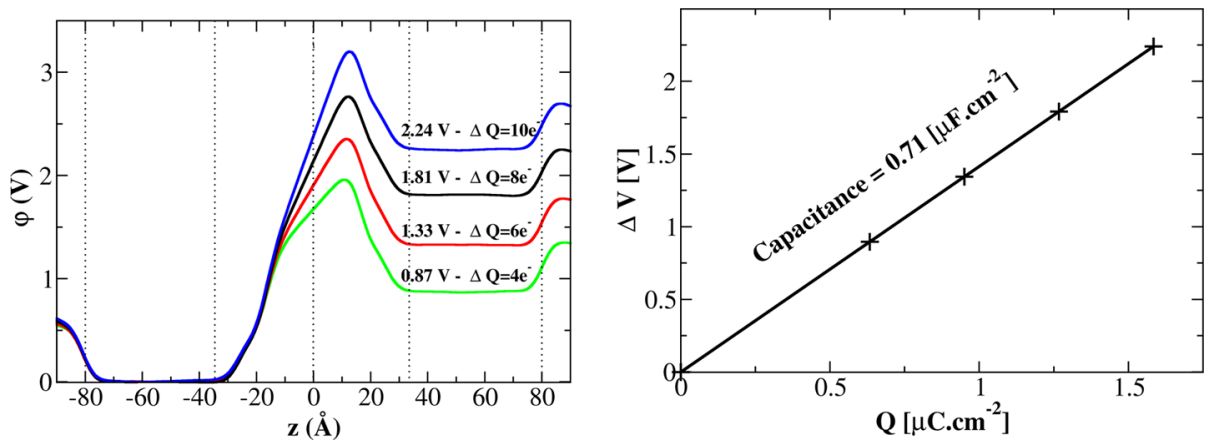

Figure 1. (Left) Electrostatic potential across a POPS lipid bilayer for different net charge imbalances $\Delta Q$ between the upper and lower electrolytes from MD simulations considering the setup of Figure S1 in the SI. $\varphi(z)$ is estimated as an in-plane average of the EP distributions (see the SI for calculation details). As a reference it was set to zero in the lower electrolyte. (Right) TM potential $\Delta V$ as a function of the charge imbalance $Q$ per unit area. The capacitance $C$ of the bilayer can be derived from the slope of the curve.

observed in previous studies on POPC were not present in any of experiment on POPS planar lipid bilayers.

\section{RESULTS AND DISCUSSION}

$\mathrm{MD}$ simulations performed at different TM voltages $(\Delta Q)$ showed that, as demonstrated previously for POPC (palmitoyloleyl-phosphatidyl-choline) membranes, the POPS bilayer behaves as a capacitor (see Figure 1). Its capacitance of $\sim 0.71 \mu \mathrm{F} / \mathrm{cm}^{2}$ (see Figure 1) is $16 \%$ lower than the value found for the POPC membrane $\left(0.85 \mu \mathrm{F} / \mathrm{cm}^{2}\right)$ under similar conditions (salt concentration, FF parameters). This is consistent with the $20 \%$ decrease found experimentally (0.51 $\mu \mathrm{F} / \mathrm{cm}^{2}$ for POPC 50 and $0.41 \mu \mathrm{F} / \mathrm{cm}^{2}$ for POPS), which tends to indicate that the setup and the $\mathrm{MD}$ force field we use are reasonable for comparison between the two bilayers.

As in the case of zwitterionic membranes, the process of electroporation starts with the protrusion of water wires from either one or both sides of the membrane (see Figure 2). As far

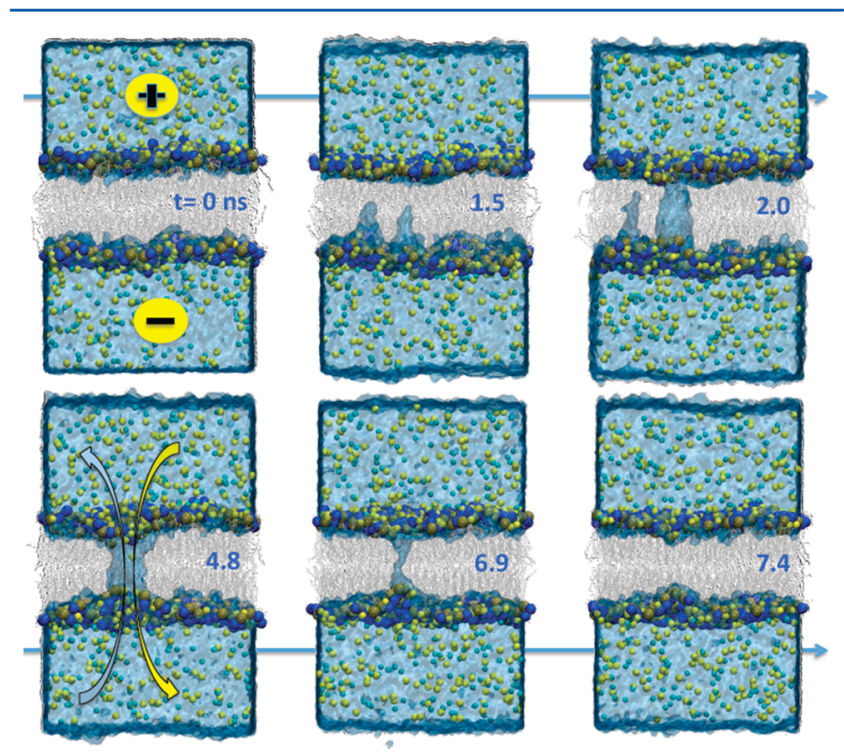

Figure 2. Snapshots along a representative MD trajectory of the formation of a hydrophobic pore within a POPS bilayer subject to a TM voltage of $\sim 4 \mathrm{~V}$. Yellow and cyan spheres depict $\mathrm{Na}^{+}$and $\mathrm{Cl}^{-}$ ions, respectively. Note that the phospholipid head groups (phosphatidyl: dark blue spheres; serine: tan spheres) remain localized at the lipid-water interface, or at the pore entrance, while water molecules (blue) flood the pore allowing for ion conduction. as only water is concerned, the latter process is reminiscent of the electric-field-induced transition from a water droplet to a water wire described by Cramer et al. ${ }^{51}$ Starting from a water droplet absorbed on a polar surface droplet, the authors demonstrated that the rise of the water pillar is induced by an electrostatic pressure that overcomes surface tension at a critical field strength. Water defects appear within a few nanoseconds (1-16 ns with a majority $\sim 5$ ns; see the SI, Table S1). This then results in the water wires joining in the membrane core. Such a defect, hereafter called a "hydrophobic pore" to indicate that the water columns are in contact with the hydrophobic lipid tails, then expands. In a few occurrences (simulations nos. 4,5 , and 12 of Table S1, SI), as in the case of the POPC, some lipid head groups dive partially into the pore to stabilize its structure ("hydrophilic" pore, see Table S1, SI). However, surprisingly, in a majority of the trajectories, the pores remained "hydrophobic" (see Figures 1 and 2, well as Figure S3 in the $\mathrm{SI}$ ). Regardless of the pore nature, ions were then driven along the electrical gradient through the pore resulting in a rapid drop of the TM voltage. Once the TM voltage reaches a few hundreds of millivolts $(0.15$ to $0.98 \mathrm{~V})$, conduction stops and the pores collapse. While full recovery of the membrane is not observed for tens of nanoseconds in the case of hydrophilic pores, ${ }^{29,32}$ resealing of the hydrophobic pores occurred on the other hand over a much shorter time scale, leading to a few nanoseconds pore lifetime. Electroporation was observed in each of the 13 simulations conducted.

To further investigate and corroborate these observations that suggest a drastic difference between the pores formed in charged (POPS) and those in zwitterionic (POPC) bilayers, we conducted experiments under current controlled conditions (linear rising current) similar to those recently reported for POPC bilayers. ${ }^{17}$

We studied bilayers prepared from POPS at $100 \mathrm{mM} \mathrm{KCl}$ concentration. Thirty-four planar bilayers were examined. The average membrane capacitance was $0.41 \pm 0.13 \mu \mathrm{F} / \mathrm{cm}^{2}, \sim 20 \%$ lower than that of POPC $\left(0.51 \mu \mathrm{F} / \mathrm{cm}^{2}\right) .{ }^{50}$ Six linear rising current signal slopes $k$ were used: $0.5,1.0,4.0,8.0,10.0$, and $20.0 \mu \mathrm{A} / \mathrm{s}$. As in previous investigations, for each system, the voltage breakdown $U_{\mathrm{br}}$ was defined as the voltage at the time $t_{\mathrm{br}}$, when an abrupt voltage drop due to lipid bilayer rupture was detected (see Figure 3 ). The values of $U_{\mathrm{br}}$ were not statistically different for the various $k$ values. The average breakdown voltage $U_{\mathrm{br}}$ was, however, much higher for POPS bilayers (398 $\pm 108 \mathrm{mV})$ then for POPC $(307 \pm 78 \mathrm{mV})$. Typical traces previously recorded for POPC are reported in Figure 4. 


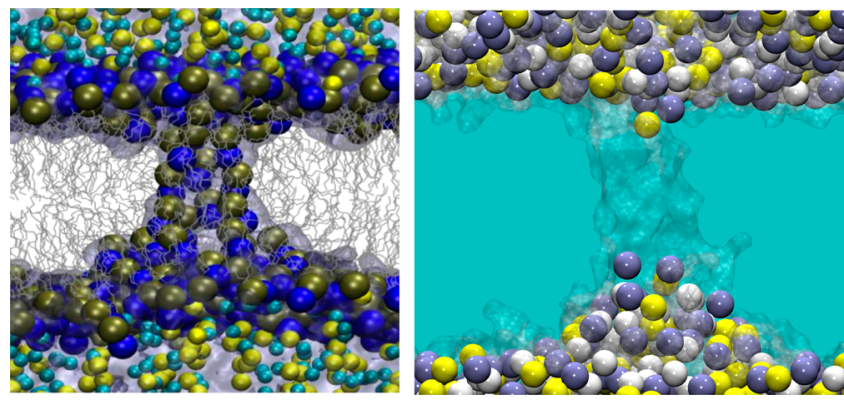

Figure 3. Snapshots of the formation of pores along representative MD trajectories within a POPS (right) and a POPC (left) bilayer subject to TM voltages leading to electroporation. Note the hydrophilic (POPC) vs hydrophobic (POPS) nature of the conducting pores. Same color code as Figure 1 .

The voltage traces present transient small voltage drops often followed by a voltage rerise within a fraction of a second. Modeling the observed phenomenon by equivalent electric circuits showed that these events relate to opening and closing of conducting pores through the bilayer. ${ }^{17}$ The most peculiar observation from the extended set of experiments on POPS bilayers performed here is that no transient voltage drop similar to those present in the POPC traces was observed in any of the 34 experiments on POPS membrane.

The high number of ion translocations monitored for each of the 13 simulations carried out in this work (see Table S1, SI) rule out the hypothesis of a low conductance pore to explain the lack of voltage drops in our experiments. In light of what has been found in the MD simulations, no detection of "drop events" in POPS bilayers may in fact be due to a recovery or resealing of pores formed as in the case of the POPC bilayers, which is too fast to be detected with actual measurement time resolution (0.01s) Accordingly, the traces of the experiments are consistent with the results from simulations.

\section{CONCLUSION}

This study shows for the first time a difference in the electroporation process between a model zwitterionic membrane and a model anionic membrane. While the initial steps of the electroporation are similar in both cases, several other parameters are different, among which an increased electroporation threshold, a hydrophobic nature of most pores formed, and a reduced lifetime of the latter. Since the 1970s the physical mechanism of electroporation (or pore creation) has been described by the Smoluchowski equation ${ }^{11}$ where it is assumed that short-lived hydrophobic pores (i.e., pore with edges formed by hydrocarbon chains of the lipids) are first created at a rate $S(t)$ that depends exponentially on the square of the transmembrane potential $\sim V$. Pores with radii $r$ above a critical radius $\left(r^{*} \approx 1 \mathrm{~nm}\right)$ convert then spontaneously to longlived stable hydrophilic pores. The overall process is governed by $S(t)$ and by the energy of the hydrophilic pore $W(r) .^{13,52}$ The possible existence of stable hydrophobic conductive pores challenges this interpretation.

Overall the present study indicates that the electroporation process is modulated by the nature of the membrane and may be different than so far assumed in "real" membranes made of a complex mixture of lipids, proteins, and sugars, possibly presenting domains with different lipid phases. Accordingly, the impact of our findings ought to be considered in biomedical applications of electroporation. On the biolotechnological front, protocols using electroporation to trigger drug-release from smart-liposome-based nanocarriers are being devised. ${ }^{53,54}$ Our study suggests that such approaches can be fine-tuned by selecting specific lipid composition, including negatively charged species.

\section{ASSOCIATED CONTENT}

\section{S Supporting Information}

Experimental details, electrostatic calculation method, and movies extracted from the MD simulations. This material is available free of charge via the Internet at http://pubs.acs.org.

\section{AUTHOR INFORMATION}

\section{Corresponding Authors}

*E-mail: francois.dehez@univ-lorraine.fr.

*E-mail: Mounir.Tarek@univ-lorraine.fr.

\section{Present Address}

${ }^{\dagger}$ Institute for Computational Molecular Science, Temple University, Philadelphia, Pennsylvania, United States.

\section{Notes}

The authors declare no competing financial interests.

\section{ACKNOWLEDGMENTS}

This work was supported by the Slovenian Research Agency and by a bilateral cooperation program between France and Slovenia (PROTEUS). This manuscript is a result of the
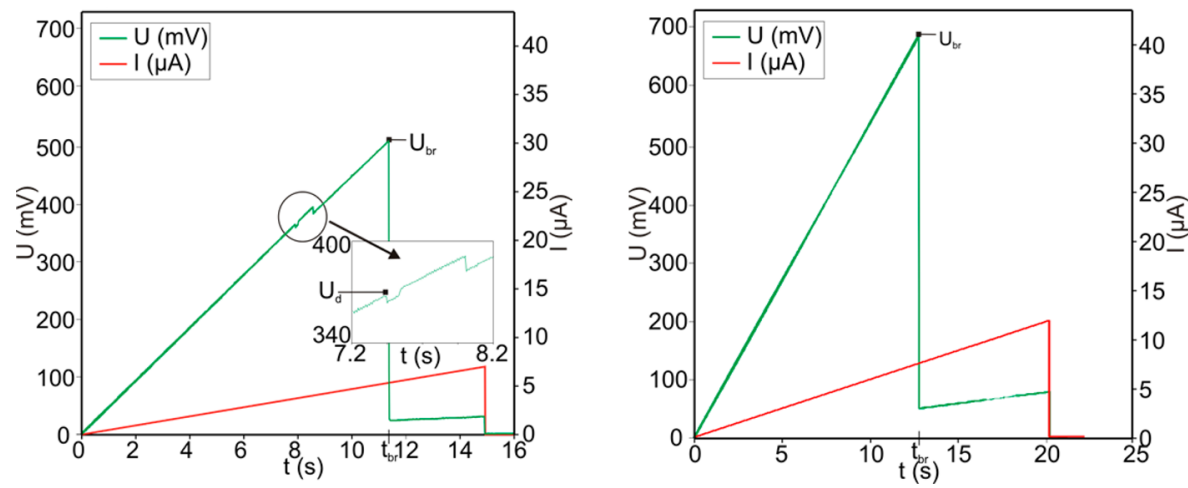

Figure 4. Typical traces of the measured TM voltage $(U)$ under linearly rising current $(I)$ conditions. (Left) POPC bilayers. ${ }^{17} U_{\mathrm{br}}$ is the voltage at which the bilayer breakdown occurs, and the arrow points at a transient voltage drop. After this event, the bilayer appears to recover completely within a fraction of a second. ${ }^{17}$ (Right) For POPS planar lipid bilayer the voltage drops are not observed in any of the measurement. 
networking efforts of the COST Action TD1104 (http://www. electroporation.net). The research was conducted in the scope of the EBAM European Associated Laboratory (LEA). Calculations were performed at the CINES, Montpellier, France, under grant no. 76434 (2010-2011). The authors thank the ANR Intcell program (ANR-10-BLAN-096).

\section{REFERENCES}

(1) Neumann, E.; Rosenheck, K. Permeability Changes Induced by Electric Impulses in Vesicular Membranes. J. Membr. Biol. 1972, 10, 279-290.

(2) Mir, L. M.; Banoun, H.; Paoletti, C. Introduction of Definite Amounts of Nonpermeant Molecules into Living Cells after Electropermeabilization: Direct Access to the Cytosol. Exp. Cell Res. 1988, 175, 15-25.

(3) Teissié, J.; Eynard, N.; Gabriel, B.; Rols, M. Electropermeabilization of Cell Membranes. Adv. Drug Delivery Rev. 1999, 35, 3-19.

(4) Golzio, M.; Teissié, J.; Rols, M.-P. Direct Visualization at the Single-Cell Level of Electrically Mediated Gene Delivery. Proc. Natl. Acad. Sci. 2002, 99, 1292-1297.

(5) Villemejane, J.; Mir, L. M. Physical Methods of Nucleic Acid Transfer: General Concepts and Applications. Br. J. Pharmacol. 2009, $157,207-219$.

(6) Mir, L. M.; Orlowski, S.; Belehradek, J., Jr.; Teissié, J.; Rols, M. P.; Serša, G.; Miklavčič, D.; Gilbert, R.; Heller, R. Biomedical Applications of Electric Pulses with Special Emphasis on Antitumor Electrochemotherapy. Bioelectrochem. Bioenerg. 1995, 38, 203-207.

(7) Serša, G.; Čemažar, M.; Miklavčič, D. Antitumor Effectiveness of Electrochemotherapy with Cis-Diamminedichloroplatinum (II) in Mice. Cancer Res. 1995, 55, 3450-3455.

(8) Heller, R.; Gilbert, R.; Jaroszeski, M. J. Clinical Applications of Electrochemotherapy. Adv. Drug Delivery Rev. 1999, 35, 119-129.

(9) Chang, D. C.; Chassy, B. M.; Saunders, J. A.; Sowers, A. E. Guide to Electroporation and Electrofusion; Academic Press: New York, NY, 1992.

(10) Weaver, J. C. Electroporation of Biological Membranes from Multicellular to Nano Scales. IEEE Trans. Dielectr. Electr. Insul. 2003, $10,754-768$.

(11) Abidor, I. G.; Arakelyan, V. B.; Chernomordik, L. V.; Chizmadzhev, Y. A.; Pastushenko, V. F.; Tarasevich, M. P. Electric Breakdown of Bilayer Lipid Membranes: I. The Main Experimental Facts and Their Qualitative Discussion. J. Electroanal. Chem. Interfacial Electrochem. 1979, 104, 37-52.

(12) Benz, R.; Beckers, F.; Zimmermann, U. Reversible Electrical Breakdown of Lipid Bilayer Membranes: A Charge-Pulse Relaxation Study. J. Membr. Biol. 1979, 48, 181-204.

(13) Weaver, J. C.; Chizmadzhev, Y. A. Theory of Electroporation: A Review. Bioelectrochem. Bioenerg. 1996, 41, 135-160.

(14) Chen, C.; Smye, S. W.; Robinson, M. P.; Evans, J. A. Membrane Electroporation Theories: A Review. Med. Biol. Eng. Comput. 2006, 44, 5-14.

(15) Tieleman, D. P.; Leontiadou, H.; Mark, A. E.; Marrink, S.-J. Simulation of Pore Formation in Lipid Bilayers by Mechanical Stress and Electric Fields. J. Am. Chem. Soc. 2003, 125, 6382-6383.

(16) Tarek, M. Membrane Electroporation: A Molecular Dynamics Simulation. Biophys. J. 2005, 88, 4045-4053.

(17) Kramar, P.; Delemotte, L.; Maček Lebar, A.; Kotulska, M.; Tarek, M.; Miklavčič, D. Molecular-Level Characterization of Lipid Membrane Electroporation Using Linearly Rising Current. J. Membr. Biol. 2012, 245, 651-659.

(18) Breton, M.; Delemotte, L.; Silve, A.; Mir, L. M.; Tarek, M. Transport of siRNA through Lipid Membranes Driven by Nanosecond Electric Pulses: An Experimental and Computational Study. J. Am. Chem. Soc. 2012, 134, 13938-13941.

(19) Ho, M.-C.; Casciola, M.; Levine, Z. A.; Vernier, P. T. Molecular Dynamics Simulations of Ion Conductance in Field-Stabilized Nanoscale Lipid Electropores. J. Phys. Chem. B 2013, 117, 1163311640 .
(20) Hu, Q.; Viswanadham, S.; Joshi, R. P.; Schoenbach, K. H.; Beebe, S. J.; Blackmore, P. F. Simulations of Transient Membrane Behavior in Cells Subjected to a High-Intensity Ultrashort Electric Pulse. Phys. Rev. E 2005, 71, 031914.

(21) Beebe, S. J.; Schoenbach, K. H. Nanosecond Pulsed Electric Fields: A New Stimulus to Activate Intracellular Signaling. J. Biomed. Biotechnol. 2005, 2005, 297-300.

(22) Vasilkoski, Z.; Esser, A. T.; Gowrishankar, T. R.; Weaver, J. C. Membrane Electroporation: The Absolute Rate Equation and Nanosecond Time Scale Pore Creation. Phys. Rev. E 2006, 74, 021904.

(23) Sundararajan, R. Nanosecond Electroporation: Another Look. Mol. Biotechnol. 2009, 41, 69-82.

(24) Deng, J.; Schoenbach, K. H.; Stephen Buescher, E.; Hair, P. S.; Fox, P. M.; Beebe, S. J. The Effects of Intense Submicrosecond Electrical Pulses on Cells. Biophys. J. 2003, 84, 2709-2714.

(25) Delemotte, L.; Tarek, M. Molecular Dynamics Simulations of Lipid Membrane Electroporation. J. Membr. Biol. 2012, 245, 531-543.

(26) Sachs, J. N.; Crozier, P. S.; Woolf, T. B. Atomistic Simulations of Biologically Realistic Transmembrane Potential Gradients. J. Chem. Phys. 2004, 121, 10847-10851.

(27) Gurtovenko, A. A.; Vattulainen, I. Pore Formation Coupled to Ion Transport through Lipid Membranes as Induced by Transmembrane Ionic Charge Imbalance: Atomistic Molecular Dynamics Study. J. Am. Chem. Soc. 2005, 127, 17570-17571.

(28) Kandasamy, S. K.; Larson, R. G. Cation and Anion Transport through Hydrophilic Pores in Lipid Bilayers. J. Chem. Phys. 2006, 125, 074901-074901-9.

(29) Gurtovenko, A. A.; Anwar, J.; Vattulainen, I. Defect-Mediated Trafficking across Cell Membranes: Insights from in Silico Modeling. Chem. Rev. 2010, 110, 6077-6103.

(30) Venturini, A.; Zerbetto, F. Dynamics of a Lipid Bilayer Induced by Electric Fields. Phys. Chem. Chem. Phys. 2011, 13, 9216-9222.

(31) Polak, A.; Bonhenry, D.; Dehez, F.; Kramar, P.; Miklavčič, D.; Tarek, M. On the Electroporation Thresholds of Lipid Bilayers: Molecular Dynamics Simulation Investigations. J. Membr. Biol. 2013, 246, 843-850.

(32) Vernier, P. T.; Sun, Y.; Gundersen, M. A. NanoelectropulseDriven Membrane Perturbation and Small Molecule Permeabilization. BMC Cell Biol. 2006, 7, 37.

(33) Vernier, P. T.; Ziegler, M. J.; Sun, Y.; Chang, W. V.; Gundersen, M. A.; Tieleman, D. P. Nanopore Formation and Phosphatidylserine Externalization in a Phospholipid Bilayer at High Transmembrane Potential. J. Am. Chem. Soc. 2006, 128, 6288-6289.

(34) Vernier, P. T.; Ziegler, M. J.; Sun, Y.; Gundersen, M. A.; Tieleman, D. P. Nanopore-Facilitated, Voltage-Driven Phosphatidylserine Translocation in Lipid Bilayers-in Cells and in Silico. Phys. Biol. 2006, 3, 233.

(35) Vernier, P. T.; Ziegler, M. J. Nanosecond Field Alignment of Head Group and Water Dipoles in Electroporating Phospholipid Bilayers. J. Phys. Chem. B 2007, 111, 12993-12996.

(36) Fernández, M. L.; Marshall, G.; Sagués, F.; Reigada, R. Structural and Kinetic Molecular Dynamics Study of Electroporation in Cholesterol-Containing Bilayers. J. Phys. Chem. B 2010, 114, 68556865.

(37) Piggot, T. J.; Holdbrook, D. A.; Khalid, S. Electroporation of the E. Coli and S. Aureus Membranes: Molecular Dynamics Simulations of Complex Bacterial Membranes. J. Phys. Chem. B 2011, 115, 1338113388.

(38) Mukhopadhyay, P.; Monticelli, L.; Tieleman, D. P. Molecular Dynamics Simulation of a Palmitoyl-Oleoyl Phosphatidylserine Bilayer with $\mathrm{Na}^{+}$Counterions and $\mathrm{NaCl}$. Biophys. J. 2004, 86, 1601-1609.

(39) Phillips, J. C.; Braun, R.; Wang, W.; Gumbart, J.; Tajkhorshid, E.; Villa, E.; Chipot, C.; Skeel, R. D.; Kalé, L.; Schulten, K. Scalable Molecular Dynamics with NAMD. J. Comput. Chem. 2005, 26, 17811802.

(40) Darden, T.; York, D.; Pedersen, L. Particle Mesh Ewald: An N. $\log (\mathrm{N})$ Method for Ewald Sums in Large Systems. J. Chem. Phys. 1993, 98, 10089. 
(41) Essmann, U.; Perera, L.; Berkowitz, M.; Darden, T.; Lee, H.; Pedersen, L. A Smooth Particle Mesh Ewald Method. J. Chem. Phys. 1995, 103, 8577-8593.

(42) Jorgensen, W. L.; Chandrasekhar, J.; Madura, J. D.; Impey, R. W.; Klein, M. L. Comparison of Simple Potential Functions for Simulating Liquid Water. J. Chem. Phys. 1983, 79, 926-935.

(43) Hénin, J.; Shinoda, W.; Klein, M. L. United-Atom Acyl Chains for CHARMM Phospholipids. J. Phys. Chem. B 2008, 112, 7008-7015.

(44) Delemotte, L.; Dehez, F.; Treptow, W.; Tarek, M. Modeling Membranes under a Transmembrane Potential. J. Phys. Chem. B 2008, 112, 5547-5550.

(45) Kramar, P.; Miklavcic, D.; Lebar, A. M. A System for the Determination of Planar Lipid Bilayer Breakdown Voltage and Its Applications. IEEE Trans. NanoBioscience 2009, 8, 132-138.

(46) Montal, M.; Mueller, P. Formation of Bimolecular Membranes from Lipid Monolayers and a Study of Their Electrical Properties. Proc. Natl. Acad. Sci. 1972, 69, 3561-3566.

(47) Kalinowski, S.; Ibron, G.; Bryl, K.; Figaszewski, Z. Chronopotentiometric Studies of Electroporation of Bilayer Lipid Membranes. Biochim. Biophys. Acta BBA, Biomembr. 1998, 1369, 204212.

(48) Kalinowski, S.; Figaszewski, Z. A Four-Electrode System for Measurement of Bilayer Lipid Membrane Capacitance. Meas. Sci. Technol. 1995, 6, 1043.

(49) Kalinowski, S.; Figaszewski, Z. A Four-Electrode PotentiostatGalvanostat for Studies of Bilayer Lipid Membranes. Meas. Sci. Technol. 1995, 6, 1050.

(50) Sabotin, I.; Lebar, A. M.; Miklavcic, D.; Kramar, P. Measurement Protocol for Planar Lipid Bilayer Viscoelastic Properties. IEEE Trans. Dielectr. Electr. Insul. 2009, 16, 1236-1242.

(51) Cramer, T.; Zerbetto, F.; García, R. Molecular Mechanism of Water Bridge Buildup: Field-Induced Formation of Nanoscale Menisci. Langmuir 2008, 24, 6116-6120.

(52) Neu, J. C.; Krassowska, W. Asymptotic Model of Electroporation. Phys. Rev. E 1999, 59, 3471-3482.

(53) ElBayoumi, T. A.; Torchilin, V. P. Current Trends in Liposome Research. In Liposomes; Weissig, V., Ed.; Methods in Molecular Biology; Humana Press: Totowa, NJ, 2010; pp 1-27.

(54) Napotnik, T. B.; Reberšek, M.; Kotnik, T.; Lebrasseur, E.; Cabodevila, G.; Miklavčič, D. Electropermeabilization of Endocytotic Vesicles in B16 F1Mouse Melanoma Cells. Med. Biol. Eng. Comput. 2010, 48, 407-413. 